VII Congresso Brasileiro de Informática na Educação (CBIE 2018)

Anais do XXIX Simpósio Brasileiro de Informática na Educação (SBIE 2018)

\title{
Detecção de Bullying Escolar em Redes Sociais e suas Implicações na Educação de Adolescentes
}

\author{
Telson Urtiga, Thais H. C. Castro \\ Instituto de Computação - Universidade Federal do Amazonas (UFAM) \\ Manaus - AM - Brazil \\ $\{$ tfcu,thais $\} @$ icomp.ufam.edu.br
}

\begin{abstract}
Online communities have been created in online social networks for different purposes. Adolescents need to belong to groups and continually seek acceptance. This often raises the issue of bullying in schools and today their most aggressive behavior in open online social networks such as twitter, facebook, tumblr and blogger, doing cyberbullying, which can lead to many cyber crimes. This work presents an investigation and implementation of a text mining model to detect bullying in social networks (cyberbullying). The standards, once identified, form a checklist with the systematization that identifies practices that led teenagers to commit cyberbullying after initiating a "social" cyberbullying process. This checklist can be made available to the school so that they become aware of the attitudes that trigger cyberbullying and cyberbullying, contributing to the preventive education of adolescents.
\end{abstract}

Resumo. Comunidades online têm sido criadas em redes sociais online para diferentes objetivos. Os adolescentes possuem necessidade de pertencerem grupos e continuamente buscarem aceitação. Isso muitas vezes gera a questão do bullying nas escolas e, hoje, sua conduta mais agressiva nas redes sociais online abertas, como twitter, facebook, tumblr e blogger, fazendo o cyberbullying, que pode levar a muitos crimes virtuais. Este trabalho apresenta uma investigação e implementação de um modelo de mineração de texto para detecção de bullying em redes sociais (cyberbullying). Os padrões, após identificados, formam um checklist com a sistematização que identifica práticas que levaram os adolescentes a cometer crimes virtuais, após terem iniciado um processo "social" de cyberbullying. Esse checklist pode ser disponilizado à escola para que fiquem em alerta sobre as atitudes que desencadeiam o cyberbullying e crimes virtuais, contribuindo para a educação preventiva dos adolescentes.

\section{Introdução}

$\mathrm{Na}$ internet, a expansão das redes sociais possibilita o desenvolvimento de um sistema colaborativo entre os indivíduos separados pela distância e pelas condições adversas do mundo contemporâneo, mas virtualmente unificados pelos mais diversos interesses comuns. Comunidades online têm sido criadas em redes sociais online para diferentes objetivos. Uma busca profunda sobre pessoas com dificuldades cognitivas sobre o uso de redes sociais online abertas, como twiter, facebook, tumblr e blogger [Castro e Lucke 2016]. Os criminosos virtuais, na maioria das vezes, se utilizam da inocência ou fragilidade dos usuários para 
proliferar mensagens, coletar informações privilegiadas, ou mesmo apenas prejudicar o outro de alguma forma, e que, muitas vezes, produz grande prejuízo moral ou financeiro para a vítima. Esses criminosos atuam de várias maneiras, e cometem crimes tais como roubo de identidade, pedofilia, calúnia e difamação, ameaça, discriminação, espionagem, etc.

A análise e extração de conhecimento de redes sociais vêm sendo amplamente utilizada em várias áreas, incluindo as ciências sociais e comportamentais, economia e marketing, onde a compreensão do comportamento da sociedade é estratégica. As redes sociais estão entre os mais complexos e importantes desafios no gerenciamento de grandes volumes de dados. Tal complexidade é justificada principalmente pela inerente interdisciplinaridade envolvida na construção dessas aplicações que integram resultados de pesquisa de disciplinas tais como Gerenciamento de Grandes Volumes de Dados, Recuperação de Informação, Visualização de Informações, Engenharia de Software, Multimídia/Hipermídia, Interação Humano-Computador, Algoritmos, Inteligência Artificial, entre outras [Cervi 2008].

Modelos de geração de grafos podem ser construídos incorporando características de redes sociais. Essas características podem ser usadas para prever como uma rede se comportará no futuro. Uma das características das redes sociais é que elas raramente são estáticas. A representação gráfica da rede social evolui à medida que os relacionamentos e as entidades são incluídos e excluídos ao longo do tempo [Han e Kamber 2006].

Tomemos como base do estudo um dos comportamentos mais latentes e danosos da sociedade, que não vem de agora, porém com o mundo virtual e suas redes sociais está cada vez mais presentes no nosso dia a dia. A palavra bullying, do inglês bully (valentão, brigão e tirano) é traduzida em português como assédio escolar, que descreve o comportamento agressivo entre estudantes. Embora sua nomeação tenha atravessado barreiras culturais, sendo usada mundialmente por pesquisadores e instalada nos dicionários como nome próprio sinalizando ações que vão além de agredir ou maltratar, ainda não há um termo em português que abarque todo o seu significado [Lopes 2011]. O bullying é um problema bastante recorrente nas escolas, sendo algumas vezes iniciado e outras ampliado nas redes sociais. Sua detecção precoce, portanto, evitaria que se tornassem crimes ou cybercrimes, além de contribuir para a intervenção na escola.

\section{Fundamentação Teórica}

Dado amplo uso das redes sociais e a grande diversidade de seus usuários, surge uma série de oportunidades a serem exploradas, entre elas o uso da mineração de dados, para descobrir padrões e prever tendências dos seus usuários. Para que seja possível analisar comportamento de indivíduos e sua evolução dentro de uma rede social é preciso que técnicas computacionais de mineração de dados sejam utilizadas. Através do conteúdo disponível nas redes sociais, nos grupos formados por adolescentes e em postagens pessoais, extrairemos informações relevantes quanto a "crimes virtuais" que sejam cometidos no ambiente das mesmas, emitindo alertas para os responsáveis e informando sobre a penalidade de tal conduta. As técnicas de análise de redes sociais atualmente suportam tais ações, mas não foram encontradas ferramentas que se ocupem em identificar grupos de adolescentes que cometem esses crimes. Partindo desse princípio e tendo em vista os vários casos de crimes cometidos por usuários diariamente, muitos até sem ter nenhum tipo de conhecimento de 
VII Congresso Brasileiro de Informática na Educação (CBIE 2018)

Anais do XXIX Simpósio Brasileiro de Informática na Educação (SBIE 2018)

infringir a lei vigente no país, este projeto irá apresentar uma abordagem dos crimes virtuais cometidos em redes sociais e suas condutas no Código Penal Brasileiro.

As redes sociais, de acordo com Marteleto (2001, p.72) são "[...] um conjunto de participantes autônomos, unindo ideias e recursos em torno de valores e interesses compartilhados".

No Brasil, a Análise de Redes Sociais (ARS) aplicadas à mídias sociais como o Twitter e o Facebook ainda é incipiente, tendo em vista que estas mídias sociais passaram a ser utilizadas apenas mais recentemente. Adicionalmente, muitas métricas, aplicadas a dados científicos - disponíveis na literatura - não são apropriadas para a extração e mineração de dados em mídias sociais. Isto se deve ao fato do conteúdo conter muitas gírias, erros ortográficos, diferentes sinais (como, por exemplo, os que identificam risos, emojis, etc.) e abreviações para representar ações e reações dos usuários.

Para recuperar tamanha quantidade de dados, e desse modo possibilitar a análise do comportamento de indivíduos e sua evolução dentro de uma rede social, é preciso que técnicas computacionais de mineração de dados sejam utilizadas [Cervi, 2008]. A análise do comportamento de indivíduos, bem como de sua trajetória em uma rede social depende de técnicas computacionais para a mineração de dados. Circunstância que possibilitou a união entre as áreas das Ciências Sociais e da Computação.

Os tipos de técnicas de Text Mining mais encontrados na literatura, são a sumarização, classificação e clustering [Wives, 2002]. A Sumarização consiste em extrair do texto um número pequeno de frases que possam resumir o assunto do documento original. São selecionadas as palavras e frases mais importantes do texto, ou conjunto de textos, a fim de não ser necessária a leitura prévia, e mesmo assim, obter a essência da mensagem [Dixon, 1997]. A Classificação também podem ser usadas para outros propósitos, como extração de informações, leitores de e-mail ou noticias eletrônicas [Wives, 2002], em conjunto a definição de classe a que um documento vai pertencer, levando em consideração as características definidas. O Clustering também é conhecido como agrupamento, ela tem como base agrupar textos em classes levando em consideração as características de cada documento, [Wives, 2002] identifica co-relacionamentos e associações entre objetos para facilitar a identificação das classes.

Cientistas da Universidade de Vermont, através de técnicas de Text Mining, descobriram uma forma de mensurar a alegria dos usuários da rede social Twitter, durante três anos foram coletadas mais de 46 bilhões de palavras escritas por 63 milhões de usuários da rede, espalhados pelo mundo. Para medir a alegria das pessoas em relação a cada palavra, os pesquisadores pagaram voluntários para votarem numa escala de 1 a 9 , sendo 1 menos alegre e 9 mais, para as 10 mil palavras mais comum da língua inglesa. Esse tipo de análise pode ser usadas como fontes de dados como jornais e site de notícias, por exemplo.

Alessandro Zanasi, professor da Universidade de Bologna, propõe, no seu artigo "Virtual Weapons for Real Wars: Text Mining for National Security", o uso das técnicas de descoberta de conhecimento em texto para monitorar atividades dos terroristas e descobrir seus nomes e grupos de envolvimento. Usando como base de dados, blogs, sites suspeitos, emails, mensagens trocadas em chatas, entre outras fontes, é possível, através de ferramentas de extração de informações, controlar atividades possivelmente terroristas [Zanasi, 2008]. 
VII Congresso Brasileiro de Informática na Educação (CBIE 2018)

Anais do XXIX Simpósio Brasileiro de Informática na Educação (SBIE 2018)

O Text Mining pode ser dividido em duas grandes fases: 1- Pré-processamento e integração dos dados não estruturados; 2- Análise estatísticas dos dados pré-processados para extrair conteúdo do texto. [Flynn, 2010].

O primeiro produto inovador é a própria análise de perfis de criminosos em formação (adolescentes) que será desenvolvido através de um estudo comparativo mais aprofundado sobre o comportamento dos usuários na utilização das redes sociais. Outro produto é a elaboração de um checklist com a sistematização de padrões que identifiquem práticas que levem a "crimes virtuais" cometidos, sem que os adolescentes percebam que estão cometendo. Isso tem um caráter sócio educativo, que pode levar esses adolescentes a aprenderem a se auto regularem.

Deboche, assédio, intimidação. Essas são algumas das atitudes relacionadas com o bullying. Esse tipo de atitude de um indivíduo, direcionada a uma vítima, infelizmente existe desde os primórdios da humanidade. Para que o bullying aconteça, é necessário que os agressores tenham contato com suas vítimas, e com as atuais facilidades de comunicação e interações virtuais, essas agressões têm alcançado números alarmantes. É o cyberbullying (ou bullying online), que abre as portas para assédio 24 horas por dia, por meio de computadores, celulares, ou outros meios que usam a Internet.

O bullying é um problema mundial, onde a agressão física ou moral repetitiva deixam marcas para o resto das vidas de quem sofre esse tipo de contato agressivo. No mundo real, o bullying pode ser verbal e também com agressões físicas. No cyberbullying, as agressões são baseadas em ameaças, deboches e invasões de privacidade. Há casos onde o agressor consegue obter senhas da vítima, acessa seus e-mails, redes sociais, e a partir disso rouba fotos (muitas vezes íntimas, podendo causar problemas gigantescos) e espalha essas informações ou comete atos ilegais usando os dados da vítima.

Um dos tipos de cyberbullying mais comuns é ridicularizar alguém sobre o seu modo de vestir, ou etnia, religião etc. Em tempos onda a intolerância está cada vez mais latente na sociedade, a prática do cyberbullying e do bullying estão cada vez mais comuns, se levarmos isso para um ambiente escolar, essas práticas aumentam em níveis consideráveis.

\section{Metodologia}

A metodologia utilizada em relação à natureza da pesquisa será mista. Quantitativa para recuperação dos dados filtrados e qualitativa por depender da análise manual e individualizada dos conjuntos de casos. O caráter do projeto é laboratorial, pois os testes serão realizados por inspeção, pela equipe do laboratório.

Como método de investigação e produção de resultados utilizou-se os métodos inerentes às próprias técnicas reconhecidas de análise de dados, sendo utilizados nesse trabalho o clustering e a sumarização. Para se utilizar adequadamente esses métodos foi formado um thesaurus, formado pelas palavras frequentes na rede social twitter, esquematizado em uma nuvem de palavras. Como ao extrair tweets vem uma quantidade muito grande, o corte foi feito para 3000 (três mil tweets), suficientes para formas uma base de análise significativa. 


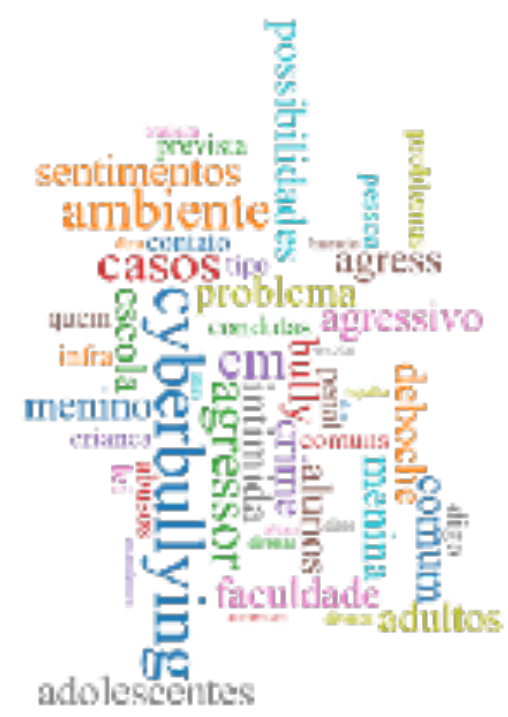

Figura 1 - Nuvem de Palavras do Twitter envolvendo Bullying

A forma de detecção dos cyberbullyings se deram de duas formas, a primeira através da extração na base de dados do twitter e analisando os tweets que continham algumas das palavras selecionadas na nuvem e seu significado, pois alguns tweets que continham as palavras não tinham o sentido do bullying em si, já outros deixavam evidente a prática do cyberbullying. A segunda forma foi através das entrevistas com adolescentes de uma turma de Ensino Médio de uma escola pública, totalizando 40 alunos, onde eles contaram boa parte da experiência, na escola e fora dela, pois muitas vezes começa na escola e depois vai para as redes sociais também, e vice versa.

\section{Monitoramento da Rede Social Twitter}

A escolha da rede social twitter se deu pelo fato de ser caracterizada por textos de até 140 caracteres. É importante salientar que a rede disponibiliza um API e esse se conecta ao software R (utilizado na coleta dos dados), tendo esse um pacote específico para rede (twitteR), entre outros pacotes para text mining, como por exemplo o $(\mathrm{tm})$. Dessa forma, o software R ou R-project foi utilizado. Ele é software livre e amplamente usado nas áreas de data mining e estatística, tendo um grande conjunto de funções pré-definidas, mas dando a liberdade de se implementar outros pacotes e funções, como por exemplo o pacote (wordcloud) que foi importante para criação da nuvem de palavras dos resultados obtidos.

Quanto às técnicas utilizadas, foram testadas várias mas utilizadas duas: o clustering e a sumarização. A técnica de clustering é conhecida também como Agrupamento, consistindo em agrupar os textos em classes de acordo com as suas características, sem a necessidade de alguma definição do usuário. Ao identificar as co-relações e associações entre os objetos o clustering busca facilitar a identificação das classes, sendo muito útil para criação de conjuntos textuais por determinado assunto, sem precisar de um conhecimento prévio do texto. [Wives 2002]. 
VII Congresso Brasileiro de Informática na Educação (CBIE 2018)

Anais do XXIX Simpósio Brasileiro de Informática na Educação (SBIE 2018)

Já a sumarização visa extrair um número pequeno de frases a fim que possa resumir o assunto do texto original. Selecionamos as palavras e frases mais importantes do texto, ou o conjunto de textos, não sendo necessária uma leitura prévia, e mesmo assim obter a essência da mensagem [Dixon 1997]. Facilitando o uso da técnica é importante que os textos a serem estudados tenham uma semelhança entre si, um exemplo, e que tenham um mesmo assunto ou tema, o que irá ajudar na hora da extração dos dados.

\subsection{Checklist}

Os padrões comportamentais após identificados, formam um checklist com a sistematização que identifica práticas que levaram os adolescentes a cometer crimes virtuais, após terem iniciado um processo "social" de cyberbullying. Muitas vezes tais comportamentos começam no ambiente escolar e passam para as redes sociais, a reciproca também é verdadeira, haja vista, que muitos se sentem "protegidos" por estarem na rede e acham que não podem sofrer punições. Esse sentimento de liberdade dos realizadores, vai de encontro ao de impunidade aos que sofrem o ataque.

Como o estudo foi dividido entre base de dados e entrevistas, tem-se um checklist para cada caso, com semelhanças em seu corpo. Na Figura 2, está parte do checklist feito para encontrar o cyberbullying cometido na rede social. Nele é possível identificar padrões nas práticas dos usuários, como por exemplo, muitos usam a hashtag \#Bullying sem nenhum tipo de pudor para realizar ataques, enquanto outros a utilizam para desabafar sobre situações vividas.

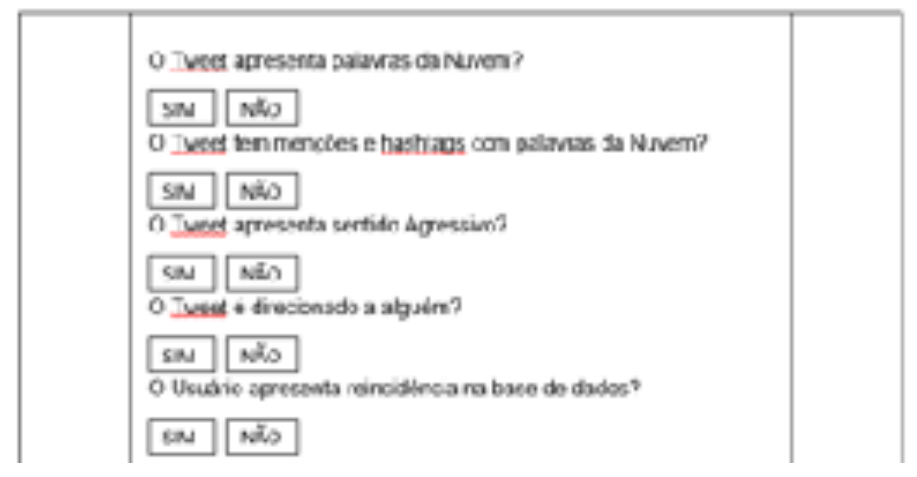

Figura 2- Parte do Checklist Rede Social

Para complementar o checklist das extrações, na entrevista com os alunos, eles mesmos puderam responder perguntas relacionadas ao tema e esclarecer algumas dúvidas a respeito. Para essa pesquisa, foi possível detectar padrões e identificar quem mais tem sofrido com o problema. Vale ressaltar que foi deixado claro, que não precisavam se identificar nas folhas, o que torna as respostas mais verdadeiras, pois em tese se elimina o quesito vergonha, amplamente difundido por quem sofre e também por quem pratica, para determinação do sexo, foi colocada um caixa com $\mathrm{M}$ ou $\mathrm{F}$, para facilitar e quantificar a análise.

A entrevista foi realizada em uma escola da rede pública de ensino, com adolescentes estudantes do Ensino Médio da mesma, com o total de 40 (quarenta) alunos envolvidos. Além do checklist, foi deixado a vontade a participação dos mesmos, para descrever casos e contar 
VII Congresso Brasileiro de Informática na Educação (CBIE 2018)

Anais do XXIX Simpósio Brasileiro de Informática na Educação (SBIE 2018)

experiências vividas por eles ou por terceiros, haja vista a quantidade significativa de acontecimentos com o termo "meu amigo sofreu...", em conjunto foi verificado se os mesmos participavam de redes sociais no geral, e na de estudo do trabalho, e se os mesmos já haviam cometido cyberbullying no Twitter, além de um espaço ao final do checklist para escritas de casos e sugestões para sanar o problema.

\section{Resultados e discussão}

De maneira estrita, um crime é uma conduta cuja realização é prevista como uma infração na legislação brasileira. Nesta perspectiva, só se poderia considerar o bullying um crime, se sua ação fosse estritamente prevista na lei. Sob essa ótica, não existe um dispositivo penal que trata especificamente sobre o bullying como algo passível de sanções. Temos um Projeto de Lei PL-1011/2011 transitando para inclusão do bullying no Código Penal Brasileiro.

Por outro lado, as condutas inerentes ao bullying geralmente são de submissão de alguém a reiteradas condições rejeitadas por essas pessoas, comportamentos agressivos de forma psicológica e física, abusos diversos e diversas maneiras de coação e coerção. Todas essas condutas são, por sua vez, consideradas infrações específicas. Em outras palavras, não existe o "crime de bullying" como um conceito fechado, mas quase todas as práticas que configuram aquilo que entendemos por bullying são infratoras do código penal. Há, portanto, punições para quem pratica, desde casos leves até prisões com duração possível de três anos.

A agressão física de um bullying, por exemplo, será julgada como lesão corporal, e é possível que os reiterados abusos no ambiente de convívio daquela pessoa sejam citados durante o processo. Em alguns casos, é possível que parte da pena inclua uma ordem de restrição para a aproximação do agressor em relação à sua vítima. Abusos de caráter psicológico também podem ser punidos através de provas de sua ocorrência e, até mesmo, de laudos médicos e psicológicos que atestem as consequências daquelas condutas agressivas no desenvolvimento de problemas para a pessoa agredida.

Alvos, autores e testemunhas enfrentam conseqüências físicas e emocionais de curto e longo prazo, as quais podem causar dificuldades acadêmicas, sociais, emocionais e legais. Evidentemente, as crianças e adolescentes não são acometidas de maneira uniforme, mas existe uma relação direta com a freqüência, duração e severidade dos atos de bullying (BOND et al.).

Pessoas que sofrem bullying quando crianças são mais propensas a sofrerem depressão e baixa autoestima quando adultos. Da mesma forma, quanto mais jovem for a criança frequentemente agressiva, maior será o risco de apresentar problemas associados a comportamentos antissociais em adultos e à perda de oportunidades, como a instabilidade no trabalho e relacionamentos afetivos pouco duradouros. O simples testemunho de atos de bullying já é suficiente para causar descontentamento com a escola e comprometimento do desenvolvimento acadêmico e social [Pearce e Thompson 1998].

A análise de perfis de criminosos em formação (adolescentes) que foi desenvolvida através de um estudo comparativo mais aprofundado sobre o comportamento dos usuários na utilização das redes sociais, levando em consideração os principais motivos que levam esses usuários a praticar tais atos, assim, podemos ter um padrão de conduta e comportamento de uma forma geral, presente na maioria dos agressores. 
VII Congresso Brasileiro de Informática na Educação (CBIE 2018)

Anais do XXIX Simpósio Brasileiro de Informática na Educação (SBIE 2018)

Com os padrões identificados, a elaboração de um checklist com a sistematização que identificam práticas que os levaram a cometer os "crimes virtuais", sem que os adolescentes percebam que estão cometendo. Querer ser mais popular, sentir-se poderoso, obter uma boa imagem de si mesmo, ter influência sobre os demais, são comportamentos padrões do agressor, levando o autor do bullying a atingir o colega com repetidas humilhações ou depreciações, até mesmo agressões físicas sendo o resultado final de uma constante prática realizada nas redes sociais. Esse agressor é uma pessoa que não aprendeu a transformar sua raiva em diálogo e para quem o sofrimento do outro não é motivo para ele deixar de agir. Pelo contrário, sente-se satisfeito com a opressão do agredido, supondo ou antecipando quão dolorosa será aquela crueldade vivida pela vítima.

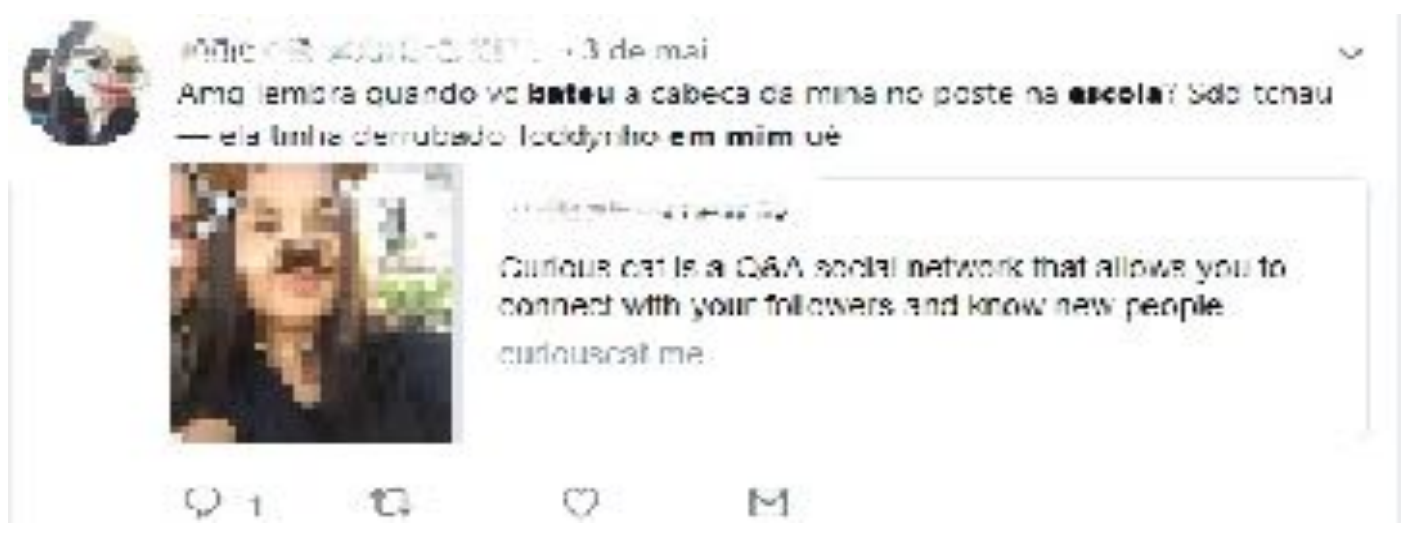

Figura 3 - Exemplo de violência praticada nas escolas expostas sem arrependimentos no Twitter

Na Figura 3, a exposição clara de uma prática relativamente comum nas escolas, uma agressão física, derivada muitas vezes de práticas de bullying, como a simples ridicularização, onde o ato de derramar a bebida pode não ter sido acidental, onde provocou uma reação de raiva, consequentemente a agressão física, podendo ser enquadrada no Código Penal, sobre Lesão Corporal, art. 129.

No estudo realizado na escola e nas bases oficiais pesquisadas, constatou-se que $57 \%$ das Meninas e $43 \%$ dos Meninos, em algum momento da sua vida escolar sofreu algum tipo de agressão, por outro lado 48\% dos Meninos e 52\% das Meninas, em algum momento praticaram agressões, assim podendo observar que os maiores agressores são do sexo feminino, como apontou a pesquisa da Unifesp em 2014. Entre os entrevistados podemos observar que o bullying tem várias formas de acontecer. Muitas vezes pequenos apelidos, insultos passam ser um tipo de agressão que muitas vezes sofrida vão ter danos irreversíveis na vida. As agressões tanto verbais quanto físicas para ser bullying não acontecem só uma vez, mas sim, repetidamente e diariamente.

Podemos então constatar (Figura 4), que o principal meio de agressão se dá verbalmente, sendo $64 \%$ entre meninos e $63 \%$ entre meninas, a surpresa fica pelo percentual de agressões físicas já que $7 \%$ das meninas afirmaram ter recebido algum tipo, enquanto $6 \%$ dos meninos fizeram a afirmação e $30 \%$ de ambos afirmaram que não sofreram nenhum tipo de agressão, como veremos no gráfico abaixo. 
VII Congresso Brasileiro de Informática na Educação (CBIE 2018)

Anais do XXIX Simpósio Brasileiro de Informática na Educação (SBIE 2018)

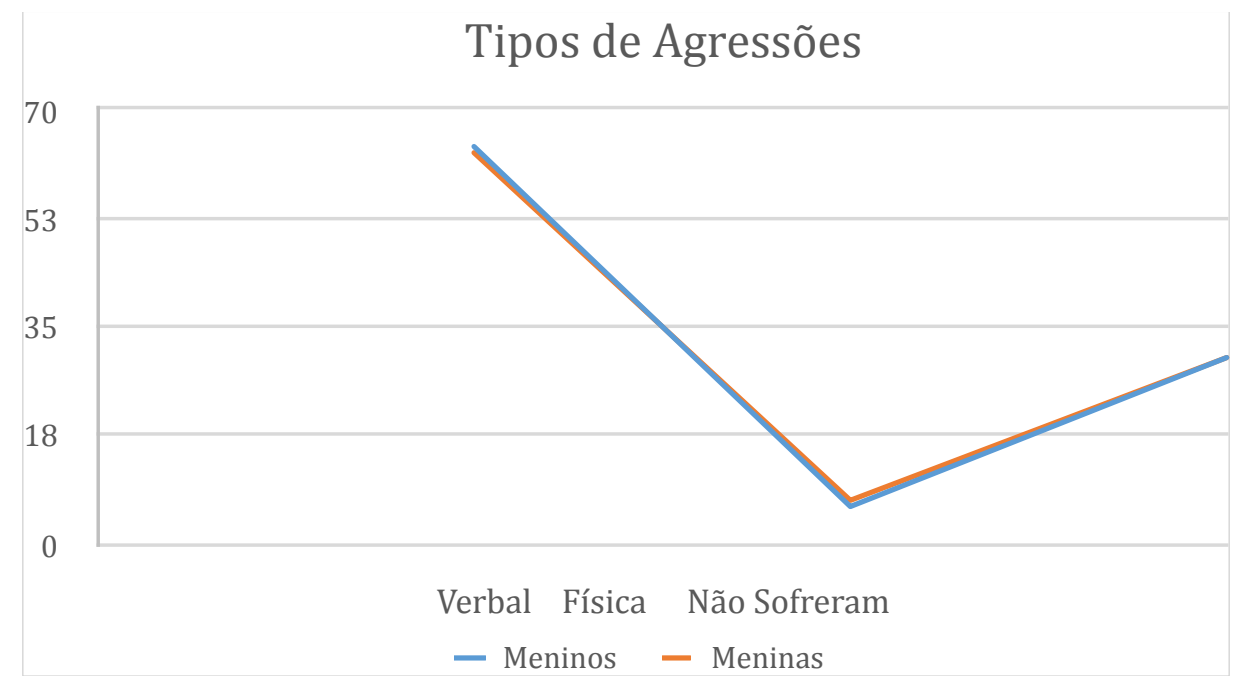

Figura 4 - Tipos de Agressões

As escolas podem aproveitar esse estudo através de um checklist organizado de acordo com os resultados obtidos tanto na análise da detecção dos cyberbullyings em si quanto nas entrevistas com os alunos: desinteresse ou a constante ausência do aluno - muitos entrevistados falaram que não tinham vontade de ir para a escola, pois sabiam que iriam ser ridicularizados; mudança no comportamento do aluno nas redes sociais - normalmente ele começa a postar menos ou para de postar e comentar; e queda no rendimento - observado pela professora presente na turma no momento das entrevistas.

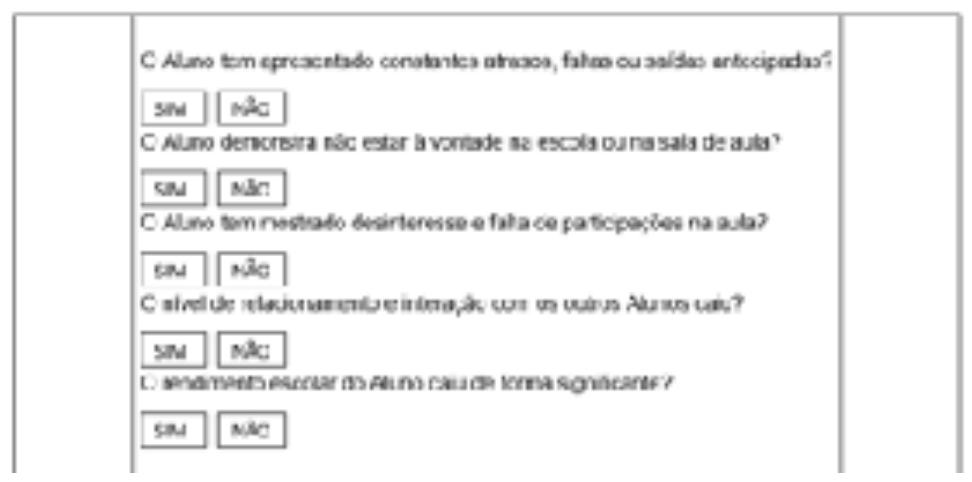

Figura 5 - Parte do Cheklist que pode ser disponibilizado as escolas

O checklist apresentado na Figura 5 pode ser disponilizado à escola para que a coordenação e o corpo docente fiquem em alerta sobre as atitudes que desencadeiam o cyberbullying e crimes virtuais, contribuindo para a educação preventiva dos adolescentes. É importante frisar que a participação dos pais e responsáveis é de suma importância, pois a maioria dos casos de cyberbullying acontecem em suas residências, logo esse controle e verificação não parte apenas da escola, a educação preventiva dos adolescentes se dá no âmbito residencial também.

\section{Considerações Finais}

As mídias sociais são recursos inovadores da contemporaneidade que precisam ser estudadas para o desvelamento das relações de atores e do compartilhamento da informação. 
VII Congresso Brasileiro de Informática na Educação (CBIE 2018)

Anais do XXIX Simpósio Brasileiro de Informática na Educação (SBIE 2018)

A funcionalidade dos aplicativos depende da mídia social da qual os dados serão analisados, cada mídia possui uma política de privacidade que limita a extração de dados. É importante, entender as limitações de cada rede antes de fazer extrações, pois pode se tornar frustrante depender de dados que não serão adquiridos e a perca com o tempo será irreversível. Buscamos apresentar de uma forma geral, a técnica de Text Mining, para obtenção de dados das redes sociais, o crescimento delas vem aumentar a necessidade de se explorar o tipo de informação nelas contidas.

Esta pesquisa também buscou apresentar um entendimento acerca de como a rede social vem sendo usada na potencialização dos chamados "crimes virtuais" e em especial o bullying que adolescentes cometem no âmbito virtual. A pesquisa partiu do princípio de que esses "crimes virtuais" cometidos em redes sociais, por meio das novas tecnologias de informação e comunicação (ciberespaço), aproveita a velocidade das informações para sua prática nessas redes, muitas vezes sem o conhecimento prévio de que se está infringindo leis, consequentemente podendo ser punido por tais atos.

Diante dos objetivos desta pesquisa verifica-se que o bullying acontece há muito tempo no âmbito escolar, se caracterizando por manifestações violentas tanto físicas quanto psicológicas, repetidamente com intenção de agredir e intimidar. Sendo assim, concluiu-se que a prática do bullying são vivenciadas em redes sociais como nas escolas, confirmando assim que esses tipos de agressões independem dos diferentes níveis sócio-econômicos, caracterizado como um reflexo que vem ocorrendo na sociedade.

\section{Referências}

Abladi S.; Weir G.; Vulnerability to Social Engineering in Social Networks: A Proposed User-Centric Framework; Glasgow, UK, 2016.

Albuquerque, Roberto Chacon de. A Criminalidade Informática. São Paulo, J. de Oliveira, 2006.

Bond L, Carlin Jb, Thomas L, Rubin K, Patton G. Does bullying cause emotional problems? A prospective study of young teenagers. BMJ. 2001;323:480-4.

Castro, T.; Lucke, U. Socialization of People with Autism Through Social Networks. In: International Conference on Universal Access in Human-Computer Interaction, 2016, Toronto. Universal Access in Human-Computer Interaction: Users and Context Diversity (Part III). Heidelberg: Springer International Publishing, 2016. v. 9. p. 193-202.

Cervi, R.; Um Estudo sobre Mineração de Dados em Redes Sociais; Porto Alegre, 2008.

Corrêa, Gustavo Testa. Aspectos Jurídicos da Internet. São Paulo: Saraiva, 2000.

Cortês, S. Da C., Porcaro, R. M., Lifschitz, S. "Mineração de Dados - Funcionalidades, Técnicas e Abordagens".PUC-Rio Inf.MCC, Rio de Janeiro, MG, 2002.

COURAGE, C. and Baxter, K. "Understanding your users: a pratical guide to user requirements, methods, tools, and techniques”. San Francisco, CA: Morgan Kaufmann Publishers, 2005. 
VII Congresso Brasileiro de Informática na Educação (CBIE 2018)

Anais do XXIX Simpósio Brasileiro de Informática na Educação (SBIE 2018)

Dixon, M. (1997). An Overview of Document Mining Technology. Disponível em $<$ http:// citeseerx.ist.psu.edu/viewdoc/download?doi=10.1.1.56.5351\&rep=rep1\&type=pdf $>$. Acesso em 29 de abr. 2016.

Feliciano, Guilherme Guimarães. Informática e Criminalidade. Ribeirão Preto: Nacional de Direito, 2001.

Flynn, M.; Francis, L. Text Mining Handbrook. Casualty Actuarial Society E-Forum, 2010.

Han J,.; Kamber M.; Data Mining: Concepts and Techniques, $2^{\text {nd }}$ ed. The Morgan Kaufmann Series in Data Management Systems, Jim Gray, Series Editor Morgan Kaufmann Publishers; 2006.

Lopes Neto A. Ações antibullying In: Lopes Neto AA. Bullying: saber identificar e como prevenir São Paulo: Brasiliense; 2011. p. 62-100.

Marteleto, R. M. "Análise de redes sociais aplicação nos estudos de transferência da informação". Programa de Pós-Graduação em Ciência da Informação, volume 30, paginas 71-81, UFRJ, Rio de Janeiro, RJ, 2001.

Meira, S. R. de L., Costa, R. A., Jucá, P. M., Silva, E. M. "Sistemas Colaborativos". Elsevier, paginas53-64, 2011.

Pang, B. and Lee, L. "Opinion Mining and Sentiment Analysis".Foundations and Trends". Volume 2, 2008.

Pearce J. B, Thompson A. C. Practical approaches to reduce the impact of bullying. Arch Dis Child. 1998;79:528-31.

Silva C.; Barbosa G.; Silva I.; Silva T.; Mourão F.; Caracterização da Usabilidade dos Recursos de Privacidade do Facebook para Crianças e Adolescentes, Revista de Informática Aplicada, Volume 12, Número 1, 2016.

Wives, L. K. Tecnologias de descoberta de conhecimento em textos aplicadas à Inteligência Competitiva. Exame de qualificação - UFRS, Porto Alegre, 2002.

Zanasi, A. Virtual Weapons for Real Wars: Text Mining for National Security. CISIS, 2008. 